CARNETS DE Carnets de géographes

GÉOGRAPHES.

$1 \mid 2010$

Varia

\title{
Sociologie du tourisme
}

Jennifer Bidet et Emmanuelle Peyvel

\section{(2) OpenEdition}

Journals

Édition électronique

URL : http://journals.openedition.org/cdg/1953

DOI : $10.4000 /$ cdg. 1953

ISSN : 2107-7266

Éditeur

UMR 245 - CESSMA

Référence électronique

Jennifer Bidet et Emmanuelle Peyvel, « Sociologie du tourisme », Carnets de géographes [En ligne], 1 । 2010, mis en ligne le 01 octobre 2010, consulté le 24 septembre 2020. URL : http:// journals.openedition.org/cdg/1953; DOI : https://doi.org/10.4000/cdg.1953

Ce document a été généré automatiquement le 24 septembre 2020.

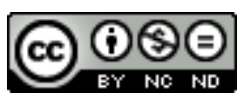

La revue Carnets de géographes est mise à disposition selon les termes de la Licence Creative Commons Attribution - Pas d'Utilisation Commerciale - Pas de Modification 4.0 International. 


\title{
Sociologie du tourisme
}

\author{
Jennifer Bidet et Emmanuelle Peyvel
}

\section{RÉFÉRENCE}

Saskia Cousin, et Bertrand Réau, 2009, Sociologie du tourisme, Paris, La Découverte, 126 p.

1 Si on ne compte plus le nombre de manuels publiés en géographie du tourisme, la sortie d'un manuel de «sociologie du tourisme » a davantage de quoi surprendre. Comme le soulignent (trop) rapidement les auteurs en introduction, l'activité touristique a longtemps été tenue à l'écart du regard de cette discipline. L'ouvrage de l'anthropologue S. Cousin et du sociologue B. Réau est alors autant un programme de recherche ouvrant la voie à de futurs travaux qu'une synthèse de ceux déjà existants. Les deux auteurs n'en sont pas à leur première entreprise pour enrichir l'étude du tourisme, ayant eux-mêmes conduit des recherches sur la question, l'une sur les politiques locales du tourisme en Touraine et l'autre sur les pratiques touristiques au Club Med. Ils sont en outre depuis 2005 à l'initiative d'un séminaire interdisciplinaire à l'acronyme évocateur «TRIP » (Tourisme Recherches Institutions Pratiques) organisé à l'EHESS.

2 Le tourisme est ici analysé pour lui-même mais surtout pour ce qu'il dit des sociétés contemporaines et de leurs mutations, "pour analyser sous un jour nouveau différents aspects des sociétés contemporaines» (p.4). La lecture des travaux s'articule autour de quatre grands questionnements servant de fil directeur à la lecture de l'ouvrage : Quels sont les déterminants sociaux et culturels des différents types de pratiques touristiques? Quelles sont les caractéristiques des métiers et des entreprises du tourisme? Comment interfèrent les sociétés locales avec le phénomène touristique ? Et quels sont les enjeux de pouvoir autour de cette activité ?

3 Le chapitre 1 (Genèses), en abordant l'invention sociale du tourisme, inscrit ces différents questionnements dans une perspective historique. Il décrit les premières formes de tourisme associées à des pratiques distinctives, relate l'apparition des premières infrastructures touristiques, évoque l'impact du développement touristique 
sur les sociétés alpines et analyse les activités de lobbying du Touring Club de France. Ce chapitre pose ainsi des jalons essentiels par rapport à la problématique de départ.

4 Le chapitre suivant (Compter et classer) s'attaque à l'épineuse question des statistiques avec lesquelles tout chercheur doit apprendre à composer. Il montre habilement combien la quantification du phénomène cristallise différentes conceptions $d u$ tourisme, fluctuant de ce fait au gré des acteurs (étatiques, commerciaux, scientifiques...), des époques, des moyens et de l'attention qu'ils lui accordent; l'actualité française étant en la matière inquiétante, puisqu'elle est placée sous le signe de la raréfaction des données faute de financements et de cohésion institutionnelle.

Le troisième chapitre (La différenciation des pratiques) aborde le tourisme du point de vue des touristes et de leurs pratiques, en tant que pratique culturelle distinctive. Au-delà des grandes tendances statistiques (soulignant l'inégal accès aux vacances), il s'agit d'identifier ce qui - qualitativement - distingue les séjours touristiques des élites des vacances populaires : si les formules peuvent être comparables (aller à la mer, etc.), la qualité de ces formules peut fortement varier et surtout les catégories les plus favorisées ont pour caractéristique de cumuler plusieurs types de séjour. Si des pratiques assez communes de vacances sont peu analysées dans les travaux existants (quid des monographies sur le camping ou quelques grandes stations balnéaires ?), en revanche les nouvelles formes du tourisme - marginales statistiquement - attirent davantage l'intérêt des chercheurs : tourisme durable, tourisme politique (à l'image de séjours au Chiapas), dark tourism (visite de camps d'extermination par exemple), tourisme "sexuel ", tourisme des racines, etc. Autant de séjours atypiques permettant de réinterroger plus largement les motivations des touristes. Le décentrement du regard permis par l'étude des touristes des pays dits du Sud vient enfin réinterroger la fausse évidence de l' «idéal touristique " en abordant les terra incognita que sont aujourd'hui pour les chercheurs les dynamiques de ces flux touristiques : alors que les touristes occidentaux sont plutôt à la recherche des traces du passé dans les pays en développement, les touristes chinois par exemple voyagent plus souvent vers les régions riches pour y contempler les symboles de la modernité.

6 Le chapitre 4 (Les marchés du tourisme) regarde le tourisme par l'autre bout de la lorgnette en s'intéressant non pas aux consommateurs mais aux producteurs du tourisme. La prise en compte des acteurs économiques n'est pas une innovation dans un secteur souvent étudié pour ses retombées économiques. L'intérêt du chapitre est ici justement de ne pas s'en tenir aux résultats globaux de cette activité (comme cela est souvent fait) mais d'aller voir en détail ses différents acteurs, gros ou petits. Les auteurs présentent ainsi la genèse de certaines entreprises leaders sur le marché (le Club Med, Nouvelles frontières, etc.), l'évolution récente du secteur (fortement mondialisé, avec de très faibles retombées économiques pour les pays pauvres) et les types d'emploi proposés.

7 Dans le chapitre 5 (Un enjeu politique), ce sont les enjeux politiques du tourisme qui sont analysés. En tant qu' "outil politique d'État» (p. 75), le tourisme peut en effet servir d'expression à l'unité nationale (cas du tourisme à l'époque coloniale ou de la politique touristique chinoise), être un instrument du contrôle social (des classes populaires comme des « minorités » culturelles) ou plus classiquement un outil d'aménagement du territoire.

8 Le dernier chapitre interroge les relations existant entre tourisme et mondialisation. Moins convaincant, reproduisant les travers de la polysémie du mot « mondialisation », 
il interroge à la fois l'idée d'un individu postmoderne hypermobile, d'une certaine uniformisation culturelle à travers une "mondialisation des images des grands sites, des villes mondiales et des destinations « hors sol » » (p. 94) et l'émergence d'institutions supraétatiques comme l'UNESCO.

9 Sont ainsi balayées au cours de l'ouvrage des problématiques variées, révélatrices de la fertilité actuelle des recherches sur le tourisme. Les tableaux statistiques et les encarts complètent habilement le texte, qu'il s'agisse de présentations d'acteurs (Thomas Cook, etc.), de thématiques (le tourisme "sexuel», etc.) ou d'outils (définitions et statistiques) relatifs au tourisme. La bibliographie fournie en fin d'ouvrage est intéressante pour son caractère interdisciplinaire. Toutefois, elle peut laisser sur sa faim concernant les études sociologiques proprement dites. Cet état de fait montre combien la sociologie du tourisme est encore nettement en construction. Le statut de l'objet « tourisme » en sociologie n'est peut-être pas ici suffisamment interrogé, en tout cas beaucoup moins que dans le numéro d'Actes de la Recherche en Sciences Sociales consacré aux «nouvelles frontières du tourisme » coordonné par les mêmes auteurs et où les enjeux internes à une sociologie du tourisme (rapport à la commande, diversité des cadres théoriques et méthodologiques, etc.) sont plus clairement présentés. Mais un manuel Repères ne comporte que 126 pages, mises ici à profit par les auteurs dans une présentation pédagogique et nuancée, ce qui relevait d'une véritable gageure.

\section{INDEX}

Thèmes : Carnets de lectures

\section{AUTEURS}

\section{JENNIFER BIDET}

Doctorante en Sociologie Université de Lyon, Groupe de Recherche sur la Socialisation (GRS-UMR 5040) ATER à l'Université Lille 3

EMMANUELLE PEYVEL

ATER en géographie à l'ENS Lyon 